\title{
The HI hole and AGB stellar population of the Sagittarius dwarf irregular galaxy
}

\section{HST proper-motion decontamination ${ }^{\star}, \star \star$}

\author{
Y. Momany ${ }^{1,2}$, M. Clemens², L. R. Bedin ${ }^{2}$, M. Gullieuszik ${ }^{2}$, E. V. Held ${ }^{2}$, I. Saviane ${ }^{1}$, S. Zaggia ${ }^{2}$, L. Monaco ${ }^{1}$, \\ M. Montalto ${ }^{3}$, R. M. Rich ${ }^{4}$, and L. Rizzi ${ }^{5}$ \\ 1 European Southern Observatory, Alonso de Cordova 3107, Santiago, Chile \\ 2 INAF, Osservatorio Astronomico di Padova, Vicolo dell' Osservatorio 5, 35122 Padova, Italy \\ e-mail: yazan.almomany@oapd.inaf.it \\ 3 Centro de Astrofisica, Universidade do Porto, Rua das Estrelas, 4150-762, Porto, Portugal \\ 4 Division of Astronomy, University of California, 8979 Math Sciences, Los Angeles, CA 90095-1562, USA \\ 5 W. M. Keck Observatory, 65-1120 Mamalahoa Highway, Waimea, HI 96743, USA
}

Received 24 April 2014 / Accepted 21 August 2014

\begin{abstract}
Using two HST/ACS data sets that are separated by about two years has allowed us to derive the relative proper motion for the Sagittarius dwarf irregular (SagDIG) and reduce the heavy foreground Galactic contamination. The proper-motion decontaminated SagDIG catalog provides a much clearer view of the young red-supergiant and intermediate-age asymptotic giant branch populations. Previously identified carbon- and oxygen-rich star samples, which are based on narrow-band filter photometry, were complemented by membership criteria. We report identifying three Milky Way carbon-rich dwarf stars, which probably belong to the thin disk, and pointing to the high incidence of this class at low Galactic latitudes. A subgroup of four oxygen-rich candidate stars depicts a faint, red extension of the well-defined SagDIG carbon-rich sequence. The origin of these oxygen-rich candidate stars remains unclear, reflecting the uncertainty in the ratio of carbon- and oxygen-rich stars. Finally, SagDIG is a gas-rich galaxy characterized by a single large cavity in the gas disk (H I hole), which is offset by $\sim 360 \mathrm{pc}$ from the optical center of the galaxy. We nonetheless investigate the stellar feedback hypothesis by comparing the proper-motion cleaned stellar populations within the $\mathrm{H}$ I hole with appropriately selected comparison regions that have higher H I densities external to the hole. The comparison shows no significant differences. In particular, the center of the $\mathrm{H}$ I hole (and the comparison regions) lack stellar populations younger than $\sim 400 \mathrm{Myr}$, which are otherwise abundant in the inner body of the galaxy. We conclude that there is no convincing evidence that the SagDIG Hi hole is the result of stellar feedback and that gravitational and thermal instabilities in the gas are the most likely mechanism for its formation.
\end{abstract}

Key words. galaxies: dwarf - galaxies: ISM - stars: AGB and post-AGB - stars: carbon - Hertzsprung-Russell and C-M diagrams astrometry

\section{Introduction}

Dwarf irregular galaxies (dIrr) play a crucial role in the ongoing effort to understand galaxy formation and evolution. Their relatively simple structure and isolated location facilitate study of stellar evolutionary phases, in particular those related to star formation processes (Mateo 1998). Moreover, the interplay between star formation and the surrounding interstellar medium (ISM) is most evident in gas-rich dIrr. The dIrr of the Local Group $(D \lesssim 1.1 \mathrm{Mpc}$ ) have a particular place in these studies, as they offer the unique possibility of investigating the interplay between the resolved stellar populations (whose average metallicity and age can be determined), the surrounding ISM, and star forming regions. The Sagittarius dwarf irregular galaxy (also known as SagDIG or UKS 1927-177) is an excellent prototype

\footnotetext{
* Based on HST programs GO-9820 and GO-10472.

$\star \star$ Full Table 1 is only available at the CDS via anonymous ftp to cdsarc.u-strasbg.fr (130.79.128.5) or via http://cdsarc.u-strasbg.fr/viz-bin/qcat?J/A+A/572/A42
}

of a star-forming, gas-rich, low-metallicity galaxy, where such studies can be conducted.

Cook (1987; hereafter C87) provided the first CCD study of the resolved stellar populations of SagDIG, where the use of two intermediate passbands allowed him to disentangle between carbon and M-type stars. Later studies by Karachentsev et al. (1999) and Lee \& Kim (2000) refined the distance modulus estimate and, at $(m-M)_{\circ}=25.13 \pm 0.25$, SagDIG was finally confirmed as a Local Group member. Interestingly, both studies agreed that SagDIG is the most metal-poor $([\mathrm{Fe} / \mathrm{H}] \sim$ -2.8) star forming galaxy in the Local Group. Momany et al. (2002) advised that a differential reddening scenario (where young centrally concentrated main-sequence stars suffer higher reddening with respect to the older and off-center red giants) would revise the SagDIG photometric metallicity to $[\mathrm{Fe} / \mathrm{H}]=$ $-2.1 \pm 0.2$, thus placing it within the general trend of the metallicity-luminosity relation for dIrr. This value was confirmed by Saviane et al. (2002) who derived an oxygen abundance of $12+\log (\mathrm{O} / \mathrm{H})=7.26$ for the brightest $\mathrm{H}$ II region in SagDIG. 
A glimpse of the star formation history of SagDIG was revealed by the deep Hubble Space Telescope (HST) study by Momany et al. (2005). Color-magnitude diagrams reaching $m_{F 606 W} \simeq 27.5$ showed the presence of a conspicuous young ( $\lesssim 1 \mathrm{Gyr}$ ) population of main-sequence and He-burning blueloop stars. The identification of the red clump indicated the presence of intermediate (1-10 Gyr) stellar populations, while that of a small but genuinely-old ( $\gtrsim 10 \mathrm{Gyr}$ ) red horizontal branch stars proved that SagDIG, just like any other dwarf galaxy, first started forming stars a Hubble time ago. Gullieuszik et al. (2008) conducted the first near-infrared study of SagDIG, and characterized the evolved asymptotic giants and red supergiant populations.

On the other hand, the spatial distribution of $\mathrm{HI}$ in gasrich dwarf galaxies is usually clumpy, and single H I clouds (50-100 pc) are found near (but do not typically coincide) with regions of active star formation and $\mathrm{H}$ II complexes (Hodge et al. 1994; Young \& Lo 1997; Hunter et al. 2012). On a larger scale, the H I observations of gas-rich dwarf galaxies reveal a wealth of structure in the ISM; some, but not all, have expanding shells (e.g., Holmberg II; Rhode et al. 1999). Cavities in the ISM of gas-rich dwarfs are believed to originate from the combined effects of photo-ionization, stellar winds and supernovae explosions from the sequential formation of massive stars. There are factors that contribute to making cavities a long-lived feature in the ISM, namely (1) the slow solid-body rotation of the gas; (2) the low gas densities; (3) the reduced shear; and (4) the absence of spiral density waves (Stewart et al. 2000).

Young \& Lo (1997) presented high resolution VLA observations showing that the SagDIG H I content extended significantly farther out than the stellar component. Without convincing signs of rotation, the H I gas seemed to be dominated by random motions. The SagDIG H I content was divided into broad $(\sigma=$ $\left.10 \mathrm{~km} \mathrm{~s}^{-1}\right)$ and narrow $\left(\sigma=5 \mathrm{~km} \mathrm{~s}^{-1}\right)$ components. The later is found in the form of small $\left(8 \times 10^{5} M_{\odot}\right)$ clumps that are mainly in the galaxy center, whereas the broad component showed a regular distribution all over the galaxy face. This two-component structure was interpreted as the analogue of the cold/warm phase structure of the Galactic H I. Young \& Lo (1997) estimated the total H I mass of SagDIG to be $\sim 9.3 \times 10^{6} M_{\odot}$, going up to $\sim 1.3 \times 10^{7} M_{\odot}$ when including He. Thus, SagDIG seems to have maintained a large reservoir in the form of neutral gas. The H I is distributed in an almost symmetric ring surrounding a cavity (horse-shoe structure) that is, again, attributed to the combined effects of stellar winds and supernovae.

All together, the general properties of the resolved stellar populations and the HI and HII components of SagDIG are known. Nevertheless, SagDIG is projected relatively close to the Galactic center $\left(\ell=21.06^{\circ}, b=-16.29^{\circ}\right)$ implying a strong foreground Galactic contamination, attributed to the Galactic thin and thick disks, the halo, and possibly the outskirts of the Galactic bulge. This is especially true for the red stellar populations of SagDIG - young red supergiants (RSG), intermediate-age carbon stars, and old asymptotic giant branch (AGB) - which are heavily confused by the Galactic foreground contamination, preventing (i) the reconstruction of the SagDIG star formation and chemical enrichment history and (ii) the interplay between the HI distribution and the SagDIG stellar populations (in particular the red ones). For the above reasons, HST second-epoch data were obtained, enabling a differential proper motion analysis of the SagDIG and foreground field populations. In this paper we present the results of the proper-motion decontamination process of our 2005 HST SagDIG catalog, which is made available in its entirety at the CDS.

\section{Observations and data reduction}

The first-epoch data set (GO-9820 P.I. Momany) consisted of three HST orbits, dedicating one orbit to each of the $F 475 \mathrm{~W}$, $F 606 W$, and $F 814 W$ filters. Five dithered exposures of $410 \mathrm{~s}$ were obtained for each filter. The observations were concluded 2003 August 18. The programmed second-epoch data set (GO-10472 P.I. Momany) consists of a single HST orbit dedicated to observations in the $F 814 W$ filter, in order to avoid possible filter-dependent systematic errors. The second-epoch data were also divided into five exposures of $410 \mathrm{~s}$. These observations were concluded 2005 June 21 . The resulting epoch separation is $\simeq 1.85$ years.

Following the general recipes given in Anderson \& King (2000), and for deriving precise astrometric measurements and a more accurate assessment of the errors, we took particular care in designing the dithering pattern of our second-epoch images in terms of both integral and fractional-pixel offsets. We then measured the positions and fluxes for all detected stars in every $F 814 W_{-} F L T$ exposure. For this task we used library effective point-spread-functions and the software programs, all documented in Anderson \& King (2006). We then generated a master list of all the stars, and collated all the observations for a given star. As in Bedin et al. (2003), we used the best distortion corrections available (Anderson 2002 and Anderson et al. 2006) to correct the raw positions measured from the _FLT exposures.

At first, we identified a group of SagDIG members in the color-magnitude diagram, and these were adopted as reference stars. The coordinate transformations from a given image into the reference frame were derived using only these reference stars (see Bedin et al. 2003, for details). We thus ensured that the proper motion of all measured stars is relative to the bulk motion of the SagDIG members. This means that the relative motion of the SagDIG stars should, to within the measurement errors, be centered on zero. We then iteratively removed some stars that had field-star-type motions from the member list, even though their colors placed them near the fiducial SagDIG sequences.

Lastly, to minimize the influence of uncorrected distortion residuals on the transformations into the reference frame, local transformations based on the nearest $\sim 50$ well-measured SagDIG stars were used for all detected stars. After taking all these precautions into account, the position of stars with $>500 \mathrm{DN}$ in their brightest pixel (in a given single image) proved to possess an error of $<0.05 \%$ of a pixel. We note that the relatively high background level in our single images $(\sim 65 \mathrm{DN})$, and the relative youth of the ACS/WFC detectors (at 2005), resulted in a negligible astrometric effect caused by charge-transfer efficiency problems.

\section{Decontaminated catalog}

The results of this differential proper motion analysis are summarized in Fig. 1. The upper lefthand panel displays the original Momany et al. (2005) composite $m_{F 814 W},\left(m_{F 475 W}-m_{F 814 W}\right)$ color-magnitude diagram towards SagDIG. The Galactic foreground contamination is visible as two broad vertical sequences at $\left(m_{F 475 W}-m_{F 814 W}\right) \simeq 1.5$ and $\simeq 3.0$, in between which the SagDIG red giant branch, red clump, and red supergiant population are recognizable thanks to their relatively higher stellar density. The young stellar population of SagDIG (composed of main 

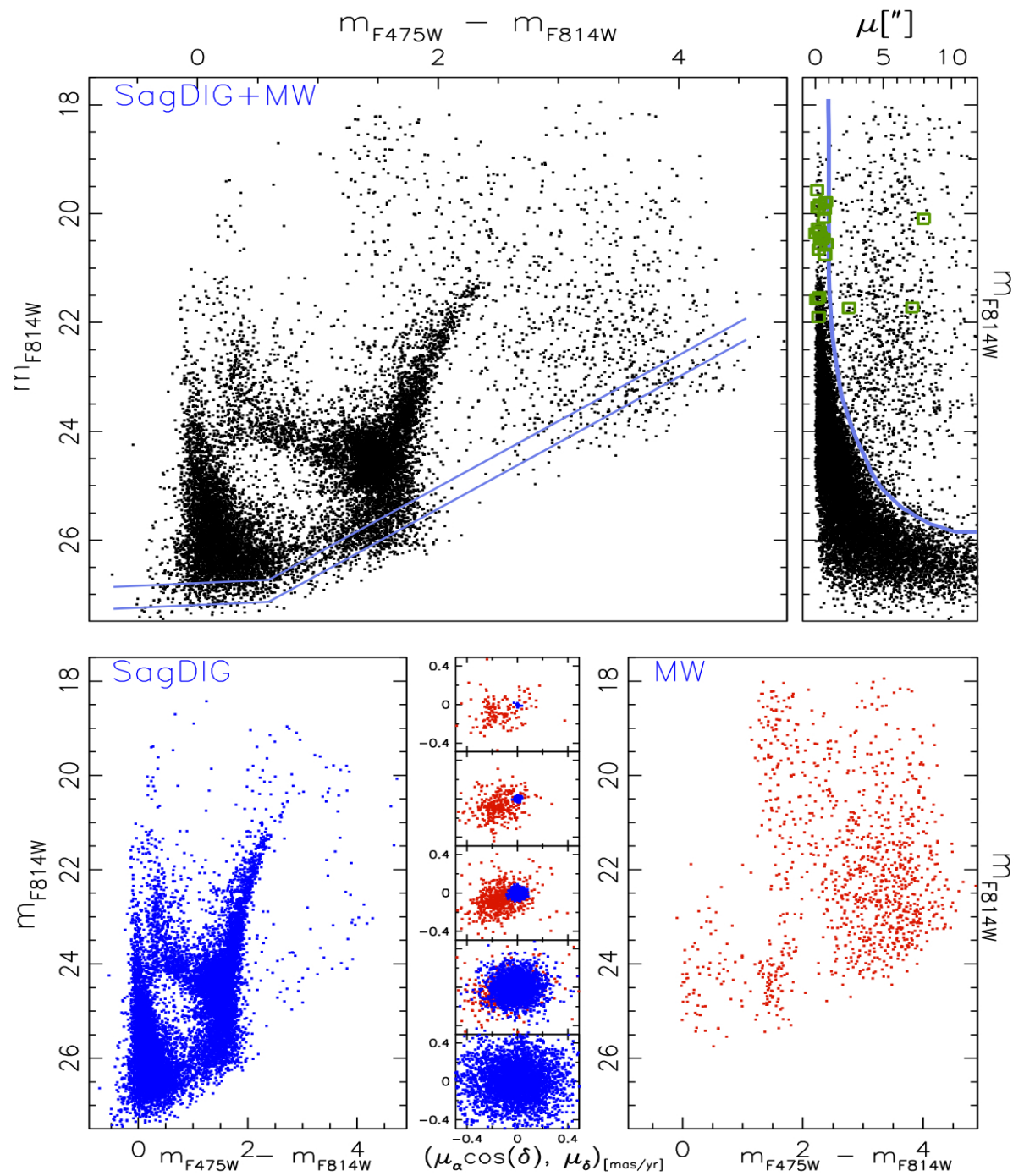

Fig. 1. Upper left panel: Momany et al. (2005) color-magnitude diagram along with the $80 \%$ and $50 \%$ photometric incompleteness levels. Upper right panel: $\mu^{\prime \prime}$ (see text) as a function of $m_{F 814 W}$, where open squares highlight the position of the identified carbon stars and the heavy blue line marks the separation between SagDIG and foreground stellar populations. Lower panels: resulting proper-motion separated diagrams of SagDIG and the Galactic foreground populations. Lower middle panel: 5 vector-point diagrams, in bins of 2.0 mag per subpanel, starting with $m_{F 606 W}=18.0$. SagDIG selected members are highlighted as blue points whereas Galactic foreground is plotted in red.

sequence and blue supergiant stars $)$ is mostly blue $\left[\left(m_{F 475 W}-\right.\right.$ $\left.\left.m_{F 814 W}\right) \lesssim 1.0\right]$, thus suffering negligible Galactic contamination. The upper righthand panel plots $\mu^{\prime \prime}$ as a function of $m_{F 814 W}$ magnitude, which is the filter in common between the data sets of the two epochs, and $\mu^{\prime \prime}$ is expressed in milliarcseconds and is calculated as $\sqrt{\mathrm{d} x^{2}+\mathrm{d} y^{2}}$ (where $\mathrm{d} x$ and $\mathrm{d} y$ are the derived offsets in pixels between the 2 epochs) normalized to the ACS pixel scale and epoch separation (i.e., $\left.\frac{\left(\sqrt{\mathrm{d} x^{2}+\mathrm{d} y^{2}} \times 0.0^{\prime} 05\right)}{1.85} \times 1000\right)$. The distribution, especially for magnitudes brighter than $m_{F 814 W} \sim$ 24.0, shows a clear separation between the SagDIG stellar populations (having $\mu^{\prime \prime} \lesssim 1.0$ ) and those belonging to the Milky Way, showing a broader $\mu^{\prime \prime} \gtrsim 2$.0. In particular, the sample of carbon stars (see next section) shows a clear separation between SagDIG members and a group of three Galactic carbon stars.

For $m_{F 814 W} \gtrsim 24.0$, increasing astrometric uncertainties do not allow a clear-cut separation between the SagDIG and Milky Way populations. Following various experiments, we conservatively draw a separating line that maximizes the number of SagDIG members. A closer look at the upper panels shows that for $m_{F 814 W} \gtrsim 24.0$ and $2.5 \lesssim\left(m_{F 475 W}-m_{F 814 W}\right) \lesssim 4.0$, the star counts of these Galactic foreground dwarfs (mostly due to the thick disk population with little contribution by the thin disk and the Galactic halo populations) drop rapidly. This is solely due to the photometric incompleteness of our first-epoch data. The thick lines in the upper lefthand diagram display the completeness levels as derived from the artificial star experiments, as presented in Momany et al. (2005).

The upper panels display the raw diagrams as a function of $m_{F 814 W}$, which was used for the differential proper motion analysis. The lower panels display the resulting cleaned diagrams, also as a function of $m_{F 814 W}$. The lower middle panel displays an enlarged view on the five vector-point diagrams, where each panel shows the distribution for bins of 2.0 mag starting $m_{F 814 W}=18.0$. The vector-point diagrams thus include the SagDIG and Galactic populations. The lower left- and righthand panels display the proper-motion decontaminated diagrams of the SagDIG and Milky Way populations, respectively.

\section{The SagDIG AGB stellar populations}

Prior to our proper-motion decontamination, the disentangling of the SagDIG red stellar populations (being RSG, oxygen and carbon-rich AGB) was hindered by the foreground Galactic contamination. To a lesser extent, and thanks to their higher number density, RGB stars allowed earlier determination of the luminosity of the tip of the giant branch. In this section we address the properties of these unveiled populations, confident that the decontamination process allows a reliable membership down to at least $F 814 W \simeq 24.0$ (i.e., the red clump level). 
A\&A 572, A42 (2014)

Table 1. SagDIG photometric ( $m_{F 475 W}, m_{F 606 W}$, and $m_{F 814 W}$ and their relative errors) and astrometric (J2000 coordinates and the pixel-based offset between the two $F 814 W$ epochs) properties of the known carbon and oxygen stars in our HST/ACS central pointing.

\begin{tabular}{|c|c|c|c|c|c|c|c|c|c|c|c|}
\hline ID & $\mathrm{RA}_{\mathrm{J} 2000}$ & Dec $_{J 2000}$ & $m_{F 475 W}$ & $e r r_{m_{F 475 W}}$ & $m_{F 606 \mathrm{~W}}$ & err. $m_{m_{F 606}}$ & $m_{F 814 W}$ & $e r r_{m_{F 814 W}}$ & $\Delta X_{\text {pixel }}$ & $\Delta Y_{\text {pixel }}$ & Type \\
\hline 1779 & 292.470424223 & -17.694280556 & 24.409 & 0.008 & 22.616 & 0.005 & 20.762 & 0.009 & 0.010 & -0.025 & carbon $[\mathrm{B} \& \mathrm{D}]$ \\
\hline 8477 & 292.484743893 & -17.679781146 & 23.825 & 0.006 & 22.212 & 0.006 & 20.454 & 0.012 & 0.008 & -0.021 & carbon $[\mathrm{B} \& \mathrm{D}]$ \\
\hline 11906 & 292.489788088 & -17.678259855 & 23.819 & 0.006 & 22.055 & 0.005 & 20.359 & 0.012 & -0.001 & 0.001 & carbon $[\mathrm{B} \& \mathrm{D}]$ \\
\hline 22018 & 292.500279743 & -17.693105356 & 24.088 & 0.010 & 21.996 & 0.007 & 20.428 & 0.010 & 0.013 & -0.005 & carbon $[\mathrm{B} \& \mathrm{D}]$ \\
\hline 28484 & 292.508378693 & -17.701596633 & 23.606 & 0.006 & 21.647 & 0.004 & 19.797 & 0.011 & 0.025 & -0.012 & carbon $[\mathrm{B} \& \mathrm{D}]$ \\
\hline 28552 & 292.506976257 & -17.685159138 & 25.278 & 0.014 & 22.784 & 0.008 & 20.658 & 0.012 & 0.005 & -0.009 & carbon $[\mathrm{B} \& \mathrm{D}]$ \\
\hline 36807 & 292.521527421 & -17.695199055 & 24.143 & 0.007 & 22.132 & 0.005 & 20.327 & 0.009 & 0.004 & -0.011 & carbon [B\&D] \\
\hline 35768 & 292.517402245 & -17.675026482 & 23.763 & 0.005 & 21.989 & 0.004 & 20.276 & 0.009 & -0.006 & -0.006 & carbon $[$ Cook+B\&D] \\
\hline 26439 & 292.504205982 & -17.681264796 & 23.578 & 0.008 & 21.657 & 0.008 & 19.883 & 0.014 & 0.006 & -0.004 & carbon $[\mathrm{Cook}+\mathrm{B} \& \mathrm{D}]$ \\
\hline 23543 & 292.501195979 & -17.684096543 & 24.799 & 0.013 & 22.175 & 0.009 & 20.069 & 0.018 & 0.010 & -0.022 & carbon $[\mathrm{Cook}+\mathrm{B} \& \mathrm{D}]$ \\
\hline 2246 & 292.470442998 & -17.675733036 & 23.773 & 0.004 & 21.775 & 0.005 & 19.923 & 0.011 & 0.001 & -0.026 & carbon $[$ Cook $+B \& D]$ \\
\hline 36305 & 292.517750836 & -17.665476415 & 23.867 & 0.004 & 22.749 & 0.004 & 21.573 & 0.006 & -0.003 & 0.002 & carbon $[$ Cook] \\
\hline 29836 & 292.50700088 & -17.667797142 & 22.797 & 0.005 & 21.100 & 0.006 & 19.570 & 0.014 & 0.005 & -0.002 & carbon [Cook] \\
\hline 26867 & 292.504035325 & -17.674187250 & 23.291 & 0.005 & 21.878 & 0.005 & 20.564 & 0.012 & 0.008 & -0.011 & carbon [Cook] \\
\hline 23532 & 292.500977236 & -17.681787246 & 23.989 & 0.010 & 22.259 & 0.008 & 20.555 & 0.011 & 0.013 & -0.028 & carbon [Cook] \\
\hline 22225 & 292.499001740 & -17.676113300 & 23.264 & 0.007 & 21.493 & 0.006 & 19.830 & 0.013 & 0.002 & -0.013 & carbon [Cook] \\
\hline 15566 & 292.494754608 & -17.685898547 & 24.029 & 0.010 & 22.948 & 0.007 & 21.903 & 0.007 & 0.006 & 0.007 & carbon [Cook] \\
\hline 6945 & 292.480575696 & -17.666244664 & 23.725 & 0.006 & 22.667 & 0.004 & 21.537 & 0.006 & 0.002 & -0.011 & carbon [Cook] \\
\hline 6090 & 292.479789823 & -17.677937700 & 23.355 & 0.005 & 21.475 & 0.007 & 19.786 & 0.015 & 0.003 & -0.030 & carbon [Cook] \\
\hline 2706 & 292.470758949 & -17.662969603 & 24.733 & 0.010 & 23.325 & 0.007 & 21.725 & 0.007 & -0.181 & -0.192 & Galactic carbon dwarf \\
\hline 22924 & 292.499538712 & -17.673751215 & 24.461 & 0.008 & 23.082 & 0.006 & 21.734 & 0.007 & -0.086 & -0.033 & Galactic carbon dwarf \\
\hline 6491 & 292.480173480 & -17.672926541 & 23.835 & 0.006 & 21.884 & 0.005 & 20.095 & 0.012 & 0.021 & -0.294 & Galactic carbon dwarf \\
\hline 35304 & 292.517603956 & -17.688463174 & 23.502 & 0.006 & 22.209 & 0.005 & 20.890 & 0.006 & 0.003 & -0.006 & oxygen $[\mathrm{B} \& \mathrm{D}]$ \\
\hline 36315 & 292.517452061 & -17.661983977 & 22.921 & 0.004 & 21.505 & 0.003 & 19.604 & 0.014 & 0.035 & -0.013 & oxygen [B\&D] \\
\hline 32223 & 292.512707052 & -17.698337255 & 25.005 & 0.009 & 23.437 & 0.006 & 21.322 & 0.006 & 0.016 & 0.011 & oxygen $[\mathrm{B} \& \mathrm{D}]$ \\
\hline 24461 & 292.504056970 & -17.704380499 & 26.152 & 0.019 & 24.327 & 0.007 & 21.478 & 0.007 & 0.021 & -0.029 & oxygen $[B \& D]$ \\
\hline 8170 & 292.482237066 & -17.658999926 & 23.050 & 0.006 & 21.691 & 0.007 & 20.224 & 0.010 & -0.011 & 0.008 & oxygen $[\mathrm{B} \& \mathrm{D}]$ \\
\hline 3971 & 292.473941125 & -17.663168733 & 25.209 & 0.014 & 23.762 & 0.008 & 21.612 & 0.010 & -0.018 & -0.027 & oxygen $[\mathrm{B} \& \mathrm{D}]$ \\
\hline 2577 & 292.470892782 & -17.668448303 & 25.747 & 0.011 & 24.278 & 0.009 & 21.856 & 0.006 & 0.007 & -0.025 & oxygen [B\&D] \\
\hline
\end{tabular}

Notes. The last column reports the identification reference. The entire SagDIG catalog is made available (in the same format as in this table) at the CDS.

Because brighter than the tip of the red giant branch, AGB stars are the first population to be detected in distant galaxies, and they have long been used (e.g., Letarte et al. 2002) to investigate the distribution of intermediate-age stellar populations. The thermally pulsating AGB evolutionary phase is characterized by mixing mechanisms that draw inner processed material to the outer stellar atmospheres. This alters the primary oxygenrich composition and gives rise to carbon-rich stars (where the ratio of CO atoms is $>1$ ). Iben \& Renzini (1983) were the first to show that the conversion from O-rich to C-rich is facilitated at lower metallicities. Indeed, in metal-poor stars, with low $\mathrm{O}$ abundance, fewer thermal pulses are required to produce $\mathrm{CO}>1$. Overall, the number ratio of $\mathrm{C}$-rich to O-rich stars allows one to infer the metallicity of a stellar population of a given age (Battinelli \& Demers 2005a). However, the CM ratio is extremely sensitive to the star formation history, since the specific production of $\mathrm{C}$ - and O-rich stars strongly depends on the initial stellar mass. This is particularly true for intermediate-mass stars for which the production of TP-AGB has a well-pronounced maximum (see Girardi et al. 2013). Intermediate-mass stars are predominately $\mathrm{C}$-rich and therefore, at a given metallicity, the CM ratio is expected to be higher in stellar systems with intermediate-age ( 1-2 Gyr) stellar populations. Consequently, this "degeneracy" has to be accounted for when the CM ratio is used to probe the metallicity of stellar populations (Sibbons et al. 2012).

One of the most efficient methods to disentangle and classify C- and O-rich stars is based on the narrow-band photometry technique pioneered by Cook et al. (1986). The method relies on the use of $\mathrm{CN}$ and $\mathrm{TiO}$ filters that are respectively centered on molecular bands present in the spectra of C-rich and O-rich stars. However, although the $\mathrm{CN}-\mathrm{TiO}$ color index is a powerful C- and O-rich separator, this technique cannot disentangle dwarf from giant stars. Consequently, when applied to select the AGB populations in nearby galaxies, control fields are used to estimate the Milky Way foreground contamination.

\subsection{Carbon-rich stars}

Cook (1987) identified a sample of 26 C stars in SagDIG. Demers \& Battinelli (2002) present another sample of C stars, eight of which are shared with the Cook sample. Of both samples, $21 \mathrm{C}$ stars were identified within our relatively smaller, HST/ACS, field of view. Table 1 presents the photometric properties of these stars, along with coordinates and proper-motion displacement. Of particular interest is the identification of three C stars whose displacement is consistent with a Galactic origin (cf. upper right panel of Fig. 1).

The upper panel of Fig. 2 shows the distribution of the $21 \mathrm{C}$ stars in the decontaminated color-magnitude diagram. The majority of the SagDIG C stars are around $\simeq 1$ mag brighter than the tip of the red giant branch, and they display redder colors. This is consistent with the $\mathrm{C}$-star identification in near-infrared diagrams (Nikolaev \& Weinberg 2000) having $(J-K) \gtrsim 1.4$, and confirmed in the near-infrared, SagDIG study of Gullieuszik et al. (2008). Battinelli \& Demers (2005b) address the standard candle aspect of carbon stars and conclude that their average $M_{I}$ luminosity is viable as long as the parent galaxy contains some hundred $\mathrm{C}$ stars or more. Indeed, their Fig. 3 shows that for large galaxies within $-20 \lesssim M_{V} \lesssim-15$, the average $M_{I}$ carbon 


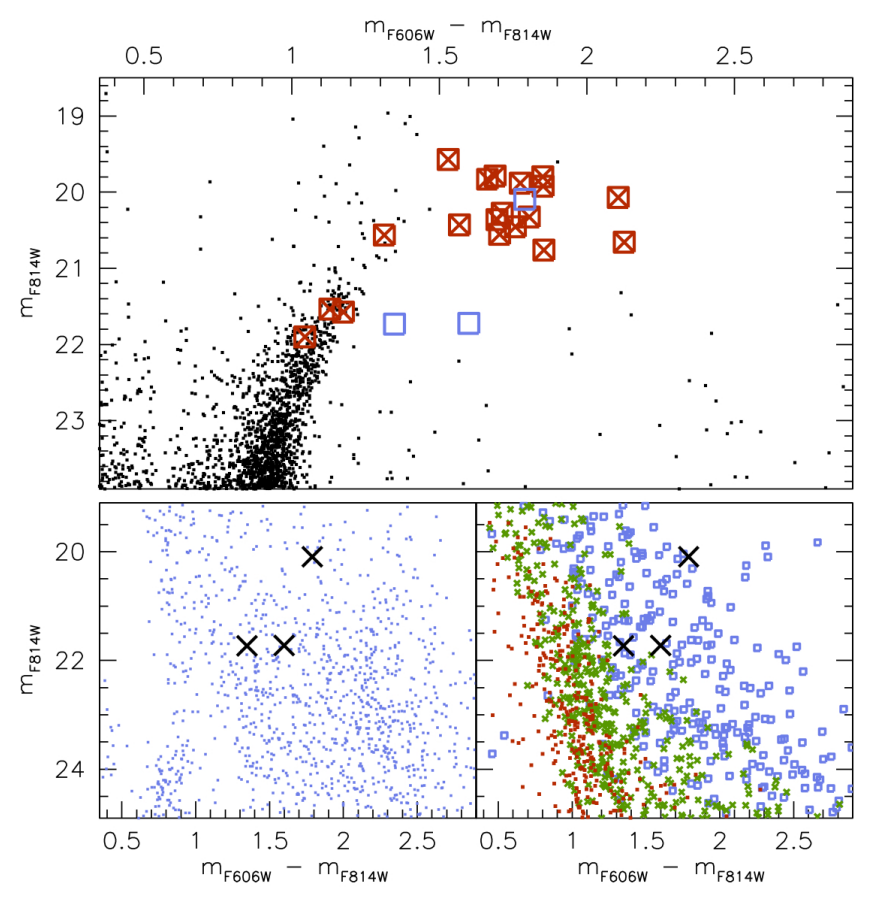

Fig. 2. Upper panel: SagDIG carbon stars highlighted on the propermotion decontaminated $m_{F 814 W},\left(m_{F 606 W}-m_{F 814 W}\right)$ color-magnitude diagram. Members are displayed as squared crosses, while those of Galactic origin are shown as open square symbols. Lower left panel: Galactic foreground proper-motion decontaminated diagram, where the 3 crosses highlight the carbon stars with Galactic membership. Lower right panel shows a Trilegal simulation of the Galactic field along the SagDIG line of sight (thin/thick disk and Galactic halo are plotted as open squares, crosses, and dots, respectively).

star luminosity is basically constant at around $-4.6 \pm 0.1$. Instead, smaller galaxies (i.e., $M_{V} \gtrsim-15$ ) show a larger dispersion. In the case of SagDIG (for which we derive $M_{V}=-11.0$ ), the average $M_{I}$ of the 16 carbon stars, which are redder than the RGB tip, is $M_{I} \simeq-4.95$, in excellent agreement with the Battinelli \& Demers (2005b) results. Our value is the result of transforming the $m_{F 814 W}$ magnitudes to $I-J o h n s o n$ Cousins systems and applying the reddening of $E_{(B-V)}=0.12$ and $(m-M) 。=25.10$ (as derived in Momany et al. 2005).

The upper panel of Fig. 2 also shows a group of three confirmed C stars clustering on the SagDIG red giant branch, just below the tip level. The identification of such faint, blue $\mathrm{C}$ stars can be evidence of binary evolution, where the red giant has accumulated carbon-processed material from the primary component of the system, now an invisible and cooling white dwarf. In this regard, the faint and blue C-star giants simply reflect C-polluted atmospheres that were produced externally, as opposed to the AGB C stars whose carbon enhancement is intrinsic to the star itself. Stellar evolutionary models can actually produce TP-AGB C stars below the red giant branch tip level (Marigo et al. 2013). The very identification of such faint, blue $\mathrm{C}$ stars would therefore indicate that the metallicity of the intermediate-age stellar populations of SagDIG is very low. This is not surprising; indeed, Saviane et al. (2002) derive a metallicity of $12+\log (\mathrm{O} / \mathrm{H})=7.26$ for the one and only $\mathrm{H}$ II region in SagDIG.

The two lower panels of Fig. 2 address the possible origin of the three Galactic C stars. The lefthand panel displays the Milky Way color-magnitude diagram as derived from the propermotion decontamination process. We compare this observed

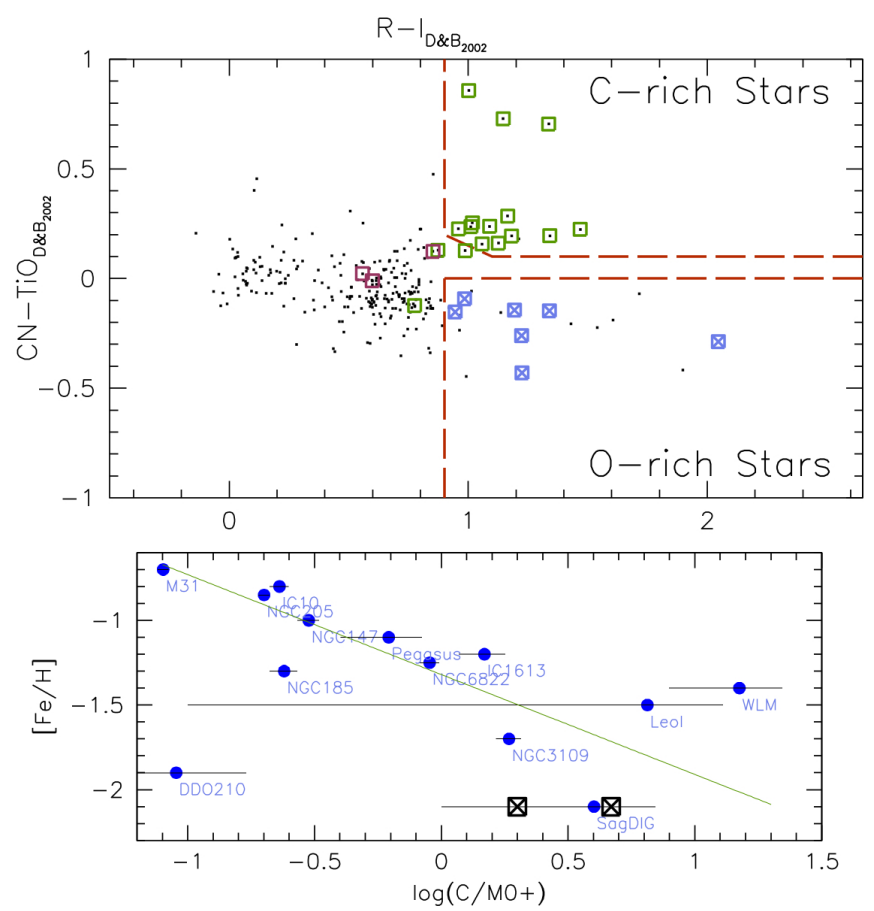

Fig. 3. Upper panel: the (CN - TiO), $(R-I)$ two-color diagram, with highlighted the Demers \& Battinelli (2002) selection boxes for carbon and oxygen-rich stars. Dark-red open squares are carbon stars (from Cook 1987) falling below the red giant branch tip. Lower panel displays a reproduction of the $[\mathrm{Fe} / \mathrm{H}], \log (\mathrm{CM} 0+)$ diagram from Battinelli \& Demers (2005b), with the new CM0+ determination for SagDIG following the proper-motion decontamination process.

diagram to a simulated diagram according to the Trilegal Milky Way model (Girardi et al. 2012) that includes thin and thick disk and halo components, all simulated in the HST/ACS photometric system. Relying solely on their position in the diagrams, the three $\mathrm{C}$ stars are consistent with being Milky Way thin disk dwarf stars. This is particularly interesting given the results from the Sloan Digital Sky Survey (Downes et al. 2004) and the Digitized First Byurakan Survey (Gigoyan et al. 2012) addressing the detection of high-latitude carbon stars. As reported in these studies, faint carbon stars were initially thought to be distant red giant branch stars. However, at least $50 \%$ of the faint carbon stars detected by the Sloan survey displayed parallaxes and/or high proper-motions that are more appropriate for nearby main-sequence stars. The relatively high proper-motion of our three $\mathrm{C}$ stars added to their position in the color-magnitude diagram all point to the first reliable detection of low-latitude Galactic carbon dwarfs, most probably belonging to the thin disk population.

\subsection{Carbon/oxygen ratio}

In this paper we adopt the CM star selection criterion following Battinelli \& Demers (2005b), which refers to the selection of stars of spectral type M0 and later, denoted as $\mathrm{M} 0+$. The $(\mathrm{CN}-\mathrm{TiO}),(R-I)$ two-color diagram (see upper panel of Fig. 3) guarantees, in principle, the identification of $C$ stars that are certain galaxy members. Indeed, the major difficulty with deriving the CM ratio resides in the selection of the oxygen-rich star sample that is foreground-contamination free. To this end, the foreground contamination is usually estimated using control fields around the galaxy of interest. In this regard, our proper-motion 


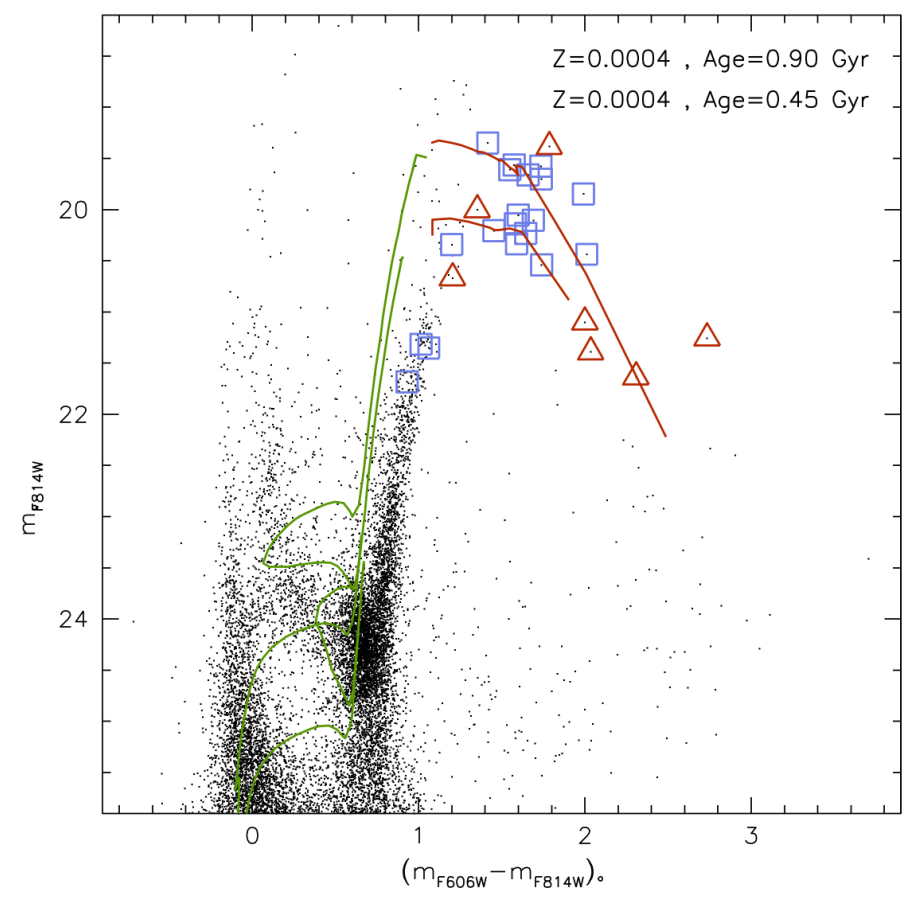

Fig. 4. Dereddened $m_{F 814 W},\left(m_{F 606 W}-m_{F 814 W}\right)$ SagDIG colormagnitude diagram highlighting the carbon stars (open squares) and 2 sets of isochrones from the Marigo et al. (2008) library. Open triangles are stars defined as oxygen-rich (see Sect. 4.2).

decontamination process provides the first independent evaluation of the $\mathrm{CM} 0+$ derivation process.

Demers \& Battinelli (2002) kindly provided us with their SagDIG 4-filter catalog and this was matched to our SagDIG member catalog, the results of which are presented in the upper panel of Fig. 3. The identification of the $\mathrm{C}$ stars is straightforward and shows the presence of a few blue C stars ${ }^{1}$ falling outside the carbon-star selection box, and includes the three carbon stars falling on the red giant branch. To ensure the selection of AGB oxygen-stars, Battinelli \& Demers (2005b) introduce another selection limit, that is, the adoption of $M_{\text {bol }}=-3.5$ for the AGB star bolometric magnitudes. We allow selection down to $\sim 0.5$ mag below the red giant branch tip, and overall count seven oxygen-rich stars vs. 14 carbon-rich stars.

The identification of the seven oxygen-rich stars (Fig. 4) warrants some attention. Indeed, four of them are very red $\left(m_{F 606 W}-m_{F 814 W} \gtrsim 1.7\right)$ and faint (with luminosities below the red giant branch tip level; $m_{F 814 W}=21.3$ ). Interestingly, the four red/faint O-rich stars appear to lie on the redder/fainter elongation of the carbon star isochrones. This hints at the possibility that these are O-rich, dust-enshrouded stars with high bolometric luminosity, and therefore one would expect them to be relatively bright at infrared wavelengths. To explore this possibility, we re-examined the SagDIG near-IR photometry from Gullieuszik et al. (2007). The four red/faint O-rich stars have $K_{\mathrm{s}}$ magnitudes that are fainter than the SagDIG RGB tip (at $\left.K_{\mathrm{s}} \sim 18.5\right)$ and $J-K_{\mathrm{s}}$ colors that are typical of normal RGB stars. Furthermore, no AllWISE (Cutri et al. 2014) counterparts were found within $1^{\prime \prime}$ of the center of the four rich stars. With the lack of strong emission at near $(2.2 \mu \mathrm{m})$ and mid-infrared $(3.4,4.6$, 12 , and $24 \mu \mathrm{m}$ ) wavelengths, one is led to doubt that the four red/faint O-rich candidate stars are genuine oxygen-rich stars.

\footnotetext{
1 We note that the blue C stars all belong to the Cook (1987) sample.
}

On the other hand, they may have a genuine oxygen-rich origin if we postulate that these four stars do need not to be massive. This possibility can be easily accommodated granted that these O-rich candidate stars reside in a significantly metal-poor environment, a constraint that is easily met by the very low metallicity of SagDIG $([\mathrm{Fe} / \mathrm{H}]=-2.1$ or $Z=0.00025)$. Indeed, the analysis presented in Nanni et al. (2013) (describing the $\mathrm{C} \leftrightarrows \mathrm{O}$ rich evolution during the thermal pulsating AGB) provides a framework within which the four red/faint stars are conceived as genuine O-rich stars. In particular, their case-d scenario (for a relatively massive four $M_{\odot}$ and $Z=0.001$ model star) shows how the transition from $\mathrm{C}$-rich to O-rich takes place following the ignition of the hot-bottom-burning process, where the efficiency of the $\mathrm{CN}$ cycle (depleting carbon) determines the transition to the O-rich spectral type.

Extrapolating the Nanni et al. (2013) case-d (4 $M_{\odot}, Z=$ 0.001 and $0.15 \mathrm{Gyr}$ ) scenario for the very low metallicity of SagDIG would allow for the appearance of $\sim 2-2.5 M_{\odot}, Z=$ 0.00025 and $0.45-0.90$ Gyr faint O-rich stars, along the extension of the C-rich stars (as seen in Fig. 4). Similarly, as also described in their case-e, the alternating effects of the third dredge-up (enhancing the production of carbon-rich stars) and hot-bottom-burning (enhancing the oxygen-rich stars) would reflect in multiple transitions across $\mathrm{CO}=1$, and O-rich stars can materialize along the extension of the red/faint $\mathrm{C}$-rich tail. One last alternative scenario (permitting the presence of thefour red/faint O-rich stars) concerns the possibility that these are less massive $\left(\lesssim 2 M_{\odot}\right)$, older ( $\gtrsim 1$ Gyr) AGB stars possessing intrinsically reddened $J-K_{\mathrm{S}}$ colors. In conclusion, the origin of these four red/faint oxygen-rich candidate stars remains uncertain, making them ideal targets for a spectroscopic follow-up.

The lower panel of Fig. 3 is a re-production of the Battinelli \& Demers (2005b) $[\mathrm{Fe} / \mathrm{H}]-\log (\mathrm{C} / \mathrm{M} 0+)$ anti-correlation for a sample of Local Group dwarf galaxies, where the straight line displays their least-square linear fit to the data. The squared crosses delimit the newly derived SagDIG CM0+ ratio including, or not, the four O-rich red/faint stars. Overall, both values fall within the expected error of Battinelli \& Demers (2005b), emphasizing the important role played by SagDIG (the mostmetal poor galaxy among the Local Group galaxies) in establishing the zero point of the $[\mathrm{Fe} / \mathrm{H}]-\log (\mathrm{C} / \mathrm{M} 0+)$ anti-correlation.

\subsection{Isochrone fitting of the carbon stars}

The thermally pulsating AGB phase is certainly the most poorly understood evolutionary phase. Indeed, this phase includes complex processes, such as the third-dredge up, hot bottom burning, mass loss through super winds, and molecular opacity calculation that has defied reliable inclusion of this phase in many of the available isochrone libraries. The recent study by Marigo et al. (2008) has not only provided a detailed and improved treatment of the former mentioned processes, but also calibrated their final isochrone set to known observable properties of TP-AGB stars. In particular, their isochrones are offered in several photometric systems. We therefore employ their HST/ACS set of isochrones directly on our data, and cautiously attempt the derivation of the C-stars' age and metallicity.

Figure 4 displays a set of isochrones from the Marigo et al. (2008) library upon the $m_{F 814 W},\left(m_{F 606 W}-m_{F 814 W}\right)$ colormagnitude diagram, with $\mathrm{C}$ stars highlighted. The bolometric corrections of Aringer et al. (2009) and a dust composition of $60 \%$ silicate $+40 \%$ AlOx for oxygen-rich stars, and $85 \%$ AMC and $15 \% \mathrm{SiC}$ are applied. Following the recipes by Sirianni et al. (2005) and assuming a distance modulus of $(m-M) 。 25.10$ 
and $E_{(B-V)}=0.12$, we adopt $A_{F 475 W}=0.437, A_{F 606 W}=0.335$, and $A_{F 814 W}=0.221$. Figure 4 summarizes our effort to derive the $\mathrm{C}$ stars' age and metallicity. For relatively metal-poor $Z=0.0004$ isochrones (reasonable for the SagDIG metallicity), we find that 0.45 and $0.90 \mathrm{Gyr}$ old isochrones reproduce the colors and luminosities of the $\mathrm{C}$ stars best. This particular point will be investigated further (Held et al., in prep.) when reconstructing the star formation history of SagDIG.

\section{The HI hole}

In Momany et al. (2005) we considered various possibilities for the formation of the large H I hole in the southwest of SagDIG. In particular, we estimated whether the integrated luminosity of the stars within the hole was sufficient to represent the remnant population of a cluster whose massive stars created the hole via supernovae explosions and winds (i.e., stellar feedback). The conclusion was that although the integrated light over the entire area of the hole was sufficient to represent such a population, the spatial distribution of stars within the hole shows no concentration toward the center of the H I hole and instead just displays a gradual increase in light intensity toward the optical center of SagDIG. Therefore, no evidence was found of any age-specific stellar population that is primarily distributed within the $\mathrm{H}$ I hole. Unless supernovae are approximately two orders of magnitude more effective at imparting mechanical energy to the ISM than currently thought (so that the putative remnant stellar population in the hole could go undetected), the only hope left for the supernovae driven wind hypothesis is that the hole is actually a very ancient structure formed by the supernovae of a cluster of stars that has since faded so much that they were undetected by the HST data used in Momany et al. (2005). The HST data, now cleaned for Galactic foreground contamination, allow us to investigate this (ancient structure) possibility more rigorously again and thereby test our previous Momany et al. (2005) conclusions (which were based solely on the integrated light within the hole).

At first glance (cf. Fig. 1) one might assume that the propermotion decontamination process adds little to our previous examination, since the data set remains the same. In particular, the massive and young stellar populations of SagDIG are relatively bluer than the Galactic turnoff color of low-mass and old mainsequence stars, which (projected along the line of sight) form a loose and vertical sequence around $m_{F 475 W}-m_{F 814 W} \sim 1.5$. This is, however, only true for the SagDIG (hydrogen-burning) main-sequence and (helium-burning) blue-supergiant stellar populations which are relatively robust to Galactic foreground contamination. On the other hand, the red-supergiant and AGB populations (which run parallel to the main SagDIG red giant branch) are subject to foreground contamination by old Galactic main-sequence stars (having reached or approaching their turnoff level/color).

Figure 4 illustrates this point further: examining the $\sim 0.45 \mathrm{Gyr}(Z=0.0004)$ isochrone in between $20.5 \leq m_{F 814 \mathrm{~W}} \leq$ 23.5 , one can appreciate how the red-supergiant population of SagDIG is outnumbered by the blue supergiants. In particular, the red-supergiant and young AGB population have such low frequency that their numbers can be easily veiled even by a low foreground contamination. Most importantly, the $\sim 0.90 \mathrm{Gyr}$ isochrone in Fig. 4 is a reminder of how identification of a handful of red-supergiant and young AGB stars (proper-motion confirmed members) would allow us to trace older stellar clusters within the hole. Indeed, detection of a few red supergiant/AGB stars at $m_{F 814 W} \sim 21.0$ would trace the presence of a

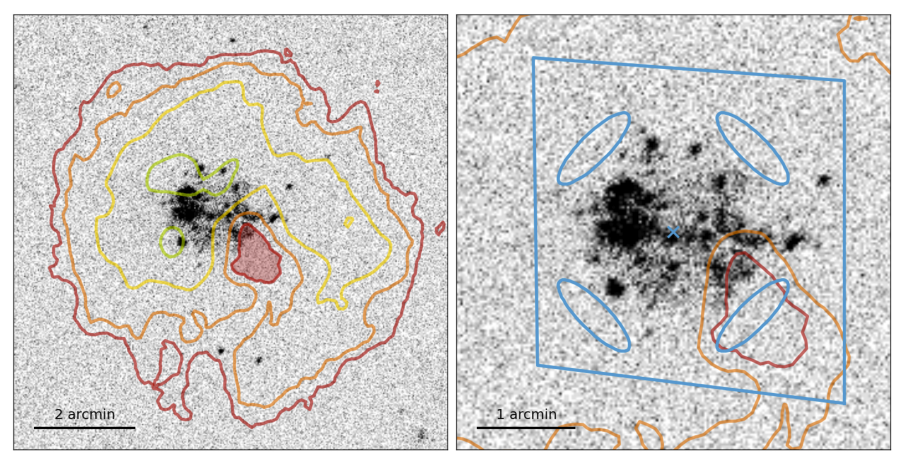

Fig. 5. GALEX far-ultraviolet image of SagDIG upon which the H I contours are overplotted. The 3 highest density $\mathrm{H}$ I clumps are the inner, open (green) contours, while the H I hole is highlighted as a shaded region. The right panel displays a closer view on the main body of SagDIG highlighting (i) the quadrangle HST/ACS field of view; (ii) the SagDIG derived center (see text) marked by a cross; and (iii) the 4 equidistant elliptical regions; used to extract and compare the stellar populations in the $\mathrm{H}$ I hole and the surrounding comparison fields.

stellar cluster with a turnoff level at $m_{F 814 W} \sim 26.3$ (at the detection limit of our data). Thus, the proper-motion decontamination process provides a unique and unbiased $\sim 1$ Gyr look-back time for the $\mathrm{H}$ I hole star formation history, tracing the remnant population of possible, past and defunct, clusters that might have provided a number of supernovae.

To guide us in our new analysis and to address the star formation history within the H I hole, we refer to Fig. 5, which displays the GALEX far-ultraviolet image of SagDIG upon which the Young \& Lo (1997) H I contours are superimposed. Besides highlighting the highest density H I clumps and void (H I hole), we also show the ACS footprint, which emphasizes the full coverage of the H I hole region. The SagDIG center [(RA, Dec $)=$ (292.499518, -17.680675)] was derived as the median position of MS, BSG and RSG stars (of all ages). To allow a direct comparison between the stellar populations present within the $\mathrm{H}$ I hole and appropriate comparison fields, we select four elliptical regions that are equidistant from the SagDIG center. The southwestern (SW) elliptical region (i.e., H I hole center) will be compared with areas with (i) moderate H I content (i.e., northwestern region) and, most interestingly; (ii) high H I column density regions (northeastern and southeastern ellipses) ${ }^{2}$.

The upper panels of Fig. 6 display the observed and propermotion decontaminated color-magnitude diagram of the stellar populations present in the selected southeast and southwest (H I hole center) equivalent regions. To minimize the poor statistics of the color-magnitude diagrams in the SagDIG comparison fields, the southeast, northeast, and northwest regions have been merged in order to infer a global star formation history of the comparison fields. We derived the star formation history (SFH) of the H I-hole and comparison fields by constructing synthetic color-magnitude diagrams (the lower panels) using the ZVAR simulator (G. Bertelli, priv. comm., which in turn is based on the uniform database of solar-scaled evolutionary tracks of Girardi et al. 2000) and comparing these to the observed color-magnitude diagrams using a maximum likelihood method. For these simulations, we have adopted a simple metal-enrichment law with metallicity linearly (and monotonically) increasing from $[\mathrm{Fe} / \mathrm{H}]=-2.1$ (as suggested for the old

\footnotetext{
2 The only known H II region (Strobel et al. 1991) is at the rim of the southeastern H I clump.
} 


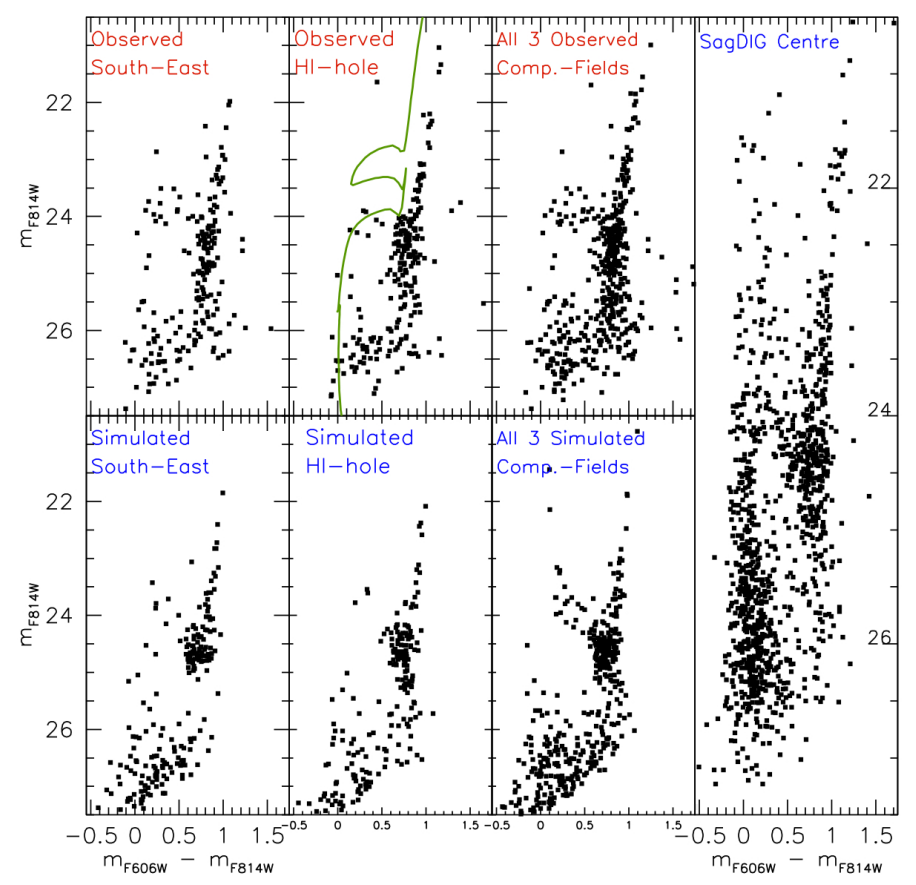

Fig. 6. From left to right: color-magnitude diagrams of the selected (see text) southeast, $\mathrm{H}$ I hole and all three comparison fields put together. The rightmost panel displays an equal-area elliptical selection around the SagDIG center, showing the abundant presence of MS and BSG stellar populations. Also plotted is a $Z=0.0004,0.355 \mathrm{Gyr}$ old isochrone.

stellar populations by our previous study Momany et al. 2005) to $[\mathrm{Fe} / \mathrm{H}]=-1.67$ (assuming for the youngest stars a metallicity similar to that of the H II region in SagDIG [1/50 solar] by Saviane et al. 2002). The present simulations do not account for internal differential reddening (which probably affects the color of the young blue plume Momany et al. 2002), assume a standard power-law initial mass function with $\alpha=-2.35$ in the interval between $0.1-100 M_{\odot}$, a binary fraction of $35 \%$, and employ the photometric errors and incompleteness levels as derived from the artificial star simulations (see Momany et al. 2005).

Although the proper-motion decontaminated SagDIG cata$\log$ is photometrically complete down to $m_{F 606 W} \simeq 26.5$, the selected stellar samples in the H I hole and comparison fields are basically void of young stars brighter than $m_{F 606 W} \simeq 23.0$. We emphasize that this null-statistics regime for stars brighter than the red clump level simply does not allow any reconstruction of the star formation history in this time interval. In particular, stellar populations younger than $\sim 350-400$ Myr can be ruled out for both the $\mathrm{H}$ I hole and the comparison fields (as is evident from the overplotted isochrones in Fig. 6). The shortage of young ( $\leq 400 \mathrm{Myr}$ ) hydrogen and helium-burning stellar populations in the selected elliptical regions is, however, fully explained by their distance from the center of SagDIG and star formation sites, which hosts abundant MS and BSG stellar populations. This is clearly illustrated in the rightmost panel of Fig. 6. A reconstruction of the recent $(\leq 500 \mathrm{Myr})$ star formation history of the center of SagDIG and an assessment of the MS, BSG, and RSG populations will be thoroughly analyzed in a dedicated paper (Held et al., in prep.).

The good agreement between the observed and simulated diagrams for stars fainter than $m_{F 606 W} \simeq 23.0$ allows us to infer that the $\mathrm{HI}$ hole and the comparison fields are similarly characterized by the onset of significant star formation between 400-800 Myr ago for stars basically populating the red clump.
In conclusion, the comparison between observed and simulated diagrams provides a quantitative confirmation of the absence of (hydrogen and helium-burning) stellar populations younger than $\sim 400$ Myr that is greater than the estimated age of the SagDIG H I hole ( 100 Myr, Momany et al. 2005) and the one in other dwarf galaxies (see Table 3 in Warren et al. 2011).

Young, $\lesssim 10$ Myr, OB associations have been traditionally (Weaver et al. 1977 and Brinks \& Bajaja 1986) associated with H I hole (shells/bubble) formation. The total absence of OB associations in the center of the $\sim 0.65 \mathrm{kpc}$ diameter SagDIG H I hole marks a distinctive difference with galaxies (e.g. Kim et al. 1999; and Hatzidimitriou et al. 2005 for the Magellanic Clouds; and Weisz et al. 2009 for Holmberg II) displaying multiple, small $\mathrm{H}$ I holes; where OB associations were detected within $\mathrm{H}$ I holes. However, the classical feedback theory (and the search for a single-age cluster) hardly provided a clear-cut, undisputed, oneto-one correspondence between the $\mathrm{OB}$ associations and the formation of $\mathrm{HI}$ holes. Indeed, cases where Hi holes ( $\sim 60 \mathrm{pc}$ diameter and $\lesssim 10$ Myr) had no associated stellar component (at all) are not rare; reaching a frequency of $\sim 10 \%$ in the case of the Small Magellanic Cloud (Hatzidimitriou et al. 2005). Moreover, the same study also established a factor of $\sim 1.5$ overabundance of H I holes that are not spatially correlated with an OB association compared to $\mathrm{H}$ I holes that are, and surprisingly infer similar properties for both $\mathrm{H}$ I hole groups.

Despite all the reported difficulties, the classical stellar feedback hypothesis remains appealing for the creation of $\mathrm{H}$ I holes and shaping of the ISM. Recently, a fine-tuning of this hypothesis has been investigated (e.g., Weisz et al. 2009; McQuinn et al. 2010; Warren et al. 2011; Cannon et al. 2011, 2012) because the search for a "single-age" stellar cluster within H I holes (by means of estimated integrated light) has been proved to be a fairly ambiguous concept given the diversity of stellar populations of different ages that are often detected within such H I holes. In particular, if allowing stellar feedback to include mixed-age stellar populations, there is the possibility that the re-occurrence of multiple supernovae (spread over hundreds of Myr) may delay the cooling of the $\mathrm{H}$ I gas, extending over the lifetime of the H I hole to $~ 0.5$ Gyr (Recchi \& Hensler 2006). Evidence of this is found in the study of McQuinn et al. (2010), who reconstruct the star formation histories of 20 dwarf galaxies and estimate that the duration of the starburst is indeed around 0.55 Gyr for two-thirds of their sample. Of particular interest is the study of Warren et al. (2011), who present a detailed analysis of five nearby dwarf irregulars whose H I morphology is dominated by a centrally dominant hole. Following the reconstruction of the star formation histories in the HI holes for each of their galaxies, they then model and quantify the stellar feedback energy (chemical, spectrophotometric, and stellar energy evolution) and compare it with the estimated energy needed to create the H I holes. Their results clearly show that the integrated stellar energy far exceeds that for the creation of the holes. Could stellar feedback from multiple age populations, extending to $\sim 0.5 \mathrm{Gyr}$, save the supernovae hypothesis in SagDIG?

At the beginning of this section we emphasized how the proper-motion decontamination process would allow us to further explore the possibility that the $\mathrm{H}$ I hole is an ancient structure, older than the traditionally assumed age ( 100-200 Myr). Indeed, given the lack of a stellar population younger than 300-400 Myr, we have focused our attention on the possible detection of a handful of bright $\left(m_{F 814 W} \sim 21.0\right) \mathrm{AGB} / \mathrm{red}$ supergiant stars (redder and distinguishable from the old red giant branch) that would trace the presence of $a \sim 1 \mathrm{Gyr}$ (now faded) cluster whose main-sequence turnoff level is about 
five magnitudes fainter. The H I hole color-magnitude diagram shows no evidence of such stars. Moreover, the same trend is seen in the three selected comparison fields, and one is led to conclude that the similarities between the stellar populations and star formation histories of the $\mathrm{H}$ I hole and control fields all point to no direct correlation between the creation of the hole and the underlying recent $(\$ 400 \mathrm{Myr})$ stellar population. Cases in which stellar feedback (also extended to include multi-age stellar populations) would have provided the necessary energy to produce and maintain the $\mathrm{HI}$ holes also (and always) infer (whenever control fields were available) that these are indistinguishable from those in the $\mathrm{H}$ I holes. Regardless of energetic arguments, it will always be difficult to attribute a hole in the atomic gas disk to the energetic input from stars if the stellar population within the hole is indistinguishable from that outside it.

We therefore abandon the hypothesis that the large H I hole in SagDIG is related to the stellar population. How then can a large hole form in the HI disk, offset from the center of the galaxy in a region that has been largely quiescent for the past $\sim 400$ Myr? The other possibilities considered in Momany et al. (2005) for its formation (top heavy IMF, much more efficient supernovae, presence of molecular gas, accreted ISM) were all found to be unlikely, but there is another possibility not considered in that article. Wada et al. (2000) show not only that kpc scale cavities can form in the ISM of dwarf galaxies, such as the LMC (with almost solid body rotation), as a natural result of thermal and gravitational instabilities, but that the dispersive effect of supernovae explosions can actually inhibit formation of large structures. Moreover, they find that although the smallscale structure of the ISM was strongly influenced by star formation (supernovae), a large-scale filamentary structure evolved even in models with no star formation at all. Such a process would allow the formation of a large H I hole in SagDIG without the need to invoke stellar feedback via supernovae.

The star formation rate in SagDIG of $2.6 \times 10^{-4} M_{\odot}$ (Momany et al. 2005) implies a supernovae rate of approximately $2.8 \times 10^{-6} M_{\odot}$. This is 400 times lower than assumed for the LMC by Wada et al. (2000). Given that the $V$-band luminosity of SagDIG is also approximately 400 times lower than the LMC $\left(M_{V}=-11.5\right.$ compared to -18.1$)$, the rate of energetic input per unit stellar mass, from supernovae into the ISM of the Sagittarius dwarf is similar to the rate in the LMC. The atomic gas mass of SagDIG, however, is only 50 times less than that of the LMC, so the energetic input per unit atomic gas mass is actually an order of magnitude (factor of $\sim 8$ ) less than in the LMC. If the effect of supernovae is actually to disrupt the formation of large-scale features in the gas, then SagDIG should represent a somewhat more favorable environment for forming large-scale features in the ISM, such as filaments and voids.

Given the lack of evidence or any age stellar population that is primarily distributed within the H I hole and the fact that SagDIG is very isolated (so that there is no obvious source from which gas may have been acquired externally), we conclude that the passive formation of a large cavity via gravitational and thermal instabilities seems to be the most likely scenario for the formation of the offset H I hole in SagDIG.

If gas disks with little differential rotation, associated with very quiescent stellar systems, are the ideal environments for the formation and growth of filamentary structure via the intrinsic instability of the ISM, then it may help explain otherwise poorly understood atomic gas morphology in such systems. One such system may be the Phoenix dwarf galaxy. In this system the atomic gas is entirely concentrated in a curved cloud offset by $590 \mathrm{pc}$ to the west of the stellar population. In fact, if we were to remove the lowest two contours from the H I map of SagDIG, it would look very similar to that of Phoenix. Young et al. (2007) suggest that this offset $\mathrm{HI}$ is caused by supernovae winds (ram pressure being excluded for lack of an extragalactic medium) from an episode of star formation that took place 100 Myr ago, but supernovae-driven winds tend to result in a symmetrical gas distribution, above and below the disk, in contrast to what is observed. A gas disk that tends to spontaneously form filamentary structure on large scales where the environment is sufficiently tranquil (appearing as "holes" in maps with limited spatial resolution) may explain the enigma of these large holes.

The frequent lack of a stellar population that could be responsible for a given H I hole has prompted Warren et al. (2011) to suggest that stars alone may not be the only driving force in the creation and maintenance of $\mathrm{HI}$ holes and that "suitable local ISM conditions" must also prevail. In particular, they note that star formation in the rims of the large $\mathrm{HI}$ holes may regulate their formation. In this regard we emphasize that the SagDIG H I hole, unlike most of those studied by Warren et al. (2011), is offset by $\sim 360 \mathrm{pc}$ from the optical center of the galaxy and any recent star formation episodes (cf. Fig. 5). In the context of an intrinsic instability in the gas, it is likely that any correlation between the border of $\mathrm{HI}$ holes and $\mathrm{H}$ II regions (if present) is simply a result of star formation being promoted in regions of greater gas density, that is, that gas morphology drives the star formation distribution and not the other way round.

\section{Summary and conclusions}

A stellar proper-motion study (based on 2-epoch HST/ACS data sets) has allowed us to disentangle the SagDIG stellar populations from the heavy Milky Way foreground contamination. The availability of cleaned SagDIG red stellar populations (being the young supergiants and intermediate-age asymptotic giant branch stars) permited an improved examination of the $\mathrm{H}$ I hole, whose origin in gas-rich dwarf galaxies is usually assumed to be the result of supernovae explosions (e.g., van Dyk et al. 1998 and Stewart et al. 2000). Our analysis shows that the stellar populations within the H I hole are very similar to those outside the hole, associated with two dense H I clumps. This clearly argues against the $\mathrm{H}$ I hole being the result of supernovae explosions.

As shown previously (Momany et al. 2005), the stellar population now present within the hole is consistent with the number of past supernovae required to provide the kinetic energy to the ISM to form the hole. However, this falls very far short of being a sufficient condition for the supernovae hypothesis. Regardless of any energetic consideration, the lack of any distinguishing stellar population within the hole, when compared to regions outside the hole, argues very strongly against the hole being caused by energetic input from the stellar population (stellar winds and supernovae). We find the possibility that gas disks with solid-body rotation and low levels of star formation can form large-scale filamentary structure as a result of thermal and gravitational instability (Wada et al. 2000) to be an appealing alternative to the supernovae-driven wind hypothesis in SagDIG (and likely other similar objects).

Previously identified via the narrow-band filter technique, the SagDIG carbon and oxygen-rich samples have been scrutinized by the proper-motion membership criterion. Of the original 22 identified carbon stars, our analysis proves that three of them are consistent with being Milky Way thin disk dwarf stars. A population of faint carbon dwarf stars was identified in highlatitude Sloan Digital Sky Survey fields by Downes et al. (2004), who estimated that they occur with a frequency of a single 
carbon dwarf per 20 square degrees. Therefore, the identification of three Milky Way carbon dwarfs in the SagDIG HST/ACS field of view (of only $\sim 3^{\prime} \times 3^{\prime}$ ) for latitudes of $\sim-16.3^{\circ}$ is suggestive that the number density of Galactic carbon dwarfs is quite significant.

The proper-motion membership criterion also permits identifying seven O-rich stars, four of which display curiously red colors and faint luminosities. Although appealing, there is no evidence of any oxygen-rich dust enshrouded stars, and indeed these four SagDIG red/faint oxygen-rich stars do not show midinfrared emission. Their presence, however, is a reminder of our poor understanding of the thermal pulsating AGB phase and the carbon-rich to oxygen-rich transition. Overall, the SagDIG proper-motion based carbon/oxygen ratio is consistently within the Battinelli \& Demers (2013) reported error. Being the most metal-poor galaxy in the Battinelli \& Demers (2013) sample, SagDIG plays an important role in establishing the zero point of the $[\mathrm{Fe} / \mathrm{H}]-\log (\mathrm{C} / \mathrm{M} 0+)$ anti-correlation.

Acknowledgements. We warmly thank the referee for the comments that greatly improved the presentation of this paper.

\section{References}

Anderson, J. 2002, in Omega Centauri, A Unique Window into Astrophysics, eds. F. van Leeuwen, J. D. Hughes, \& G. Piotto, ASP Conf. Ser., 265, 87 Anderson, J., \& King, I. R. 2000, PASP, 112, 1360

Anderson, J., \& King, I. R. 2006, PSFs, Photometry, and Astronomy for the ACS/WFC, Tech. Rep.

Anderson, J., Bedin, L. R., Piotto, G., Yadav, R. S., \& Bellini, A. 2006, A\&A, 454, 1029

Aringer, B., Girardi, L., Nowotny, W., Marigo, P., \& Lederer, M. T. 2009, A\&A, 503,913

Battinelli, P., \& Demers, S. 2005a, A\&A, 434, 657

Battinelli, P., \& Demers, S. 2005b, A\&A, 442, 159

Battinelli, P., \& Demers, S. 2013, A\&A, 553, A93

Bedin, L. R., Piotto, G., King, I. R., \& Anderson, J. 2003, AJ, 126, 247

Brinks, E., \& Bajaja, E. 1986, A\&A, 169, 14

Cannon, J. M., Giovanelli, R., Haynes, M. P., et al. 2011, ApJ, 739, L22

Cannon, J. M., O’Leary, E. M., Weisz, D. R., et al. 2012, ApJ, 747, 122

Cook, K. H. 1987, Ph.D. Thesis, Arizona Univ., Tucson

Cook, K. H., Aaronson, M., \& Norris, J. 1986, ApJ, 305, 634

Cutri, R. M., et al. 2014, VizieR Online Data Catalog, II/328

Demers, S., \& Battinelli, P. 2002, AJ, 123, 238
Downes, R. A., Margon, B., Anderson, S. F., et al. 2004, AJ, 127, 2838

Gigoyan, K. S., Russeil, D., Mickaelian, A. M., Sarkissian, A., \& Avtandilyan, M. G. 2012, A\&A, 544, A95

Girardi, L., Bressan, A., Bertelli, G., \& Chiosi, C. 2000, A\&AS, 141, 371

Girardi, L., Barbieri, M., Groenewegen, M. A. T., et al. 2012, TRILEGAL, a TRIdimensional modeL of thE GALaxy: Status and Future, eds. A. Miglio, J. Montalbán, \& A. Noels, 165

Girardi, L., Marigo, P., Bressan, A., \& Rosenfield, P. 2013, ApJ, 777, 142

Gullieuszik, M., Rejkuba, M., Cioni, M. R., Habing, H. J., \& Held, E. V. 2007, A\&A, 475, 467

Gullieuszik, M., Greggio, L., Held, E. V., et al. 2008, A\&A, 483, L5

Hatzidimitriou, D., Stanimirovic, S., Maragoudaki, F., et al. 2005, MNRAS, 360, 1171

Held, E. V., Gullieuszik, M., Rizzi, L., et al. 2010, MNRAS, 404, 1475

Hodge, P., Strobel, N. V., \& Kennicutt, R. C. 1994, PASP, 106, 309

Hunter, D. A., Ficut-Vicas, D., Ashley, T., et al. 2012, AJ, 144, 134

Iben, Jr., I., \& Renzini, A. 1983, ARA\&A, 21, 271

Karachentsev, I., Aparicio, A., \& Makarova, L. 1999, A\&A, 352, 363

Kim, S., Dopita, M. A., Staveley-Smith, L., \& Bessell, M. S. 1999, AJ, 118, 2797

Lee, M. G., \& Kim, S. C. 2000, AJ, 119, 777

Letarte, B., Demers, S., Battinelli, P., \& Kunkel, W. E. 2002, AJ, 123, 832

Marigo, P., Girardi, L., Bressan, A., et al. 2008, A\&A, 482, 883

Marigo, P., Bressan, A., Nanni, A., Girardi, L., \& Pumo, M. L. 2013, MNRAS, 434, 488

Mateo, M. L. 1998, ARA\&A, 36, 435

McQuinn, K. B. W., Skillman, E. D., Cannon, J. M., et al. 2010, ApJ, 724, 49

Momany, Y., Held, E. V., Saviane, I., \& Rizzi, L. 2002, A\&A, 384, 393

Momany, Y., Held, E. V., Saviane, I., et al. 2005, A\&A, 439, 111

Nanni, A., Bressan, A., Marigo, P., \& Girardi, L. 2013, MNRAS, 434, 2390

Nikolaev, S., \& Weinberg, M. D. 2000, ApJ, 542, 804

Recchi, S., \& Hensler, G. 2006, A\&A, 445, L39

Rhode, K. L., Salzer, J. J., Westpfahl, D. J., \& Radice, L. A. 1999, AJ, 118, 323

Saviane, I., Rizzi, L., Held, E. V., Bresolin, F., \& Momany, Y. 2002, A\&A, 390, 59

Sibbons, L. F., Ryan, S. G., Cioni, M.-R. L., Irwin, M., \& Napiwotzki, R. 2012, A\&A, 540, A135

Sirianni, M., Jee, M. J., Benítez, N., et al. 2005, PASP, 117, 1049

Stewart, S. G., Fanelli, M. N., Byrd, G. G., et al. 2000, ApJ, 529, 201

Strobel, N. V., Hodge, P., \& Kennicutt, Jr., R. C. 1991, ApJ, 383, 148

van Dyk, S. D., Puche, D., \& Wong, T. 1998, AJ, 116, 2341

Wada, K., Spaans, M., \& Kim, S. 2000, ApJ, 540, 797

Warren, S. R., Weisz, D. R., Skillman, E. D., et al. 2011, ApJ, 738, 10

Weaver, R., McCray, R., Castor, J., Shapiro, P., \& Moore, R. 1977, ApJ, 218, 377

Weisz, D. R., Skillman, E. D., Cannon, J. M., et al. 2009, ApJ, 704, 1538

Young, L. M., \& Lo, K. Y. 1997, ApJ, 490, 710

Young, L. M., Skillman, E. D., Weisz, D. R., \& Dolphin, A. E. 2007, ApJ, 659, 331 